PROCEEDINGS OF THE

AMERICAN MATHEMATICAL SOCIETY

Volume 134, Number 5, Pages 1435-1446

S 0002-9939(05)08101-3

Article electronically published on October 13, 2005

\title{
MAPS PRESERVING NUMERICAL RANGES OF OPERATOR PRODUCTS
}

\author{
JINCHUAN HOU AND QINGHUI DI
}

(Communicated by Joseph A. Ball)

\begin{abstract}
Let $H$ be a complex Hilbert space, $B(H)$ the algebra of all bounded linear operators on $H$ and $S^{a}(H)$ the real linear space of all self-adjoint operators on $H$. We characterize the surjective maps on $B(H)$ or $S^{a}(H)$ that preserve the numerical ranges of products or Jordan triple-products of operators.
\end{abstract}

\section{INTRODUCTION}

Denote by $\mathbb{C}$ the field of complex numbers and by $\mathbb{R}$ the field of real numbers. For a Hilbert space $H,\langle\cdot, \cdot\rangle$ stands for its inner product, $\mathcal{B}(H)$ the algebra of all bounded linear operators on $H$ and $\mathcal{S}^{a}(H)$ the real linear space of all self-adjoint operators in $\mathcal{B}(H)$. For every $A \in \mathcal{B}(H)$, the numerical range of $A$ is the set $W(A)=\{\langle A x, x\rangle \mid x \in H,\|x\|=1\}$ and the numerical radius of $A$ is defined as $w(A)=\sup \{|\lambda| \mid \lambda \in W(A)\}$. A map $U$ on $H$ is called a conjugate unitary operator if $U$ is conjugate linear and $U^{*} U=U U^{*}=I$.

Numerical range of operators is a very important concept and is extensively studied in both theory and applications. Particularly, many authors have studied numerical range preserving maps on various operator algebras; see [1- 6], 9], 11, [12, [13, Chapter 5]. In this paper, we characterize surjective maps $\phi: \mathcal{B}(H) \rightarrow$ $\mathcal{B}(K)$ such that

$$
W(\phi(A) \phi(B))=W(A B) \quad \text { for all } A, B \in \mathcal{B}(H) .
$$

Here $H, K$ are two Hilbert spaces. This work is motivated by the result of L. Molnár 10, who characterized surjective maps $\phi$ on $\mathcal{B}(H)$ such that

$$
\sigma(\phi(A) \phi(B))=\sigma(A B) \text { for all } A, B \in \mathcal{B}(H) .
$$

Here $\sigma(T)$ is the spectrum of $T \in \mathcal{B}(H)$.

In Section 2, we show that a surjective map $\phi: \mathcal{B}(H) \rightarrow \mathcal{B}(K)$ satisfying (1.1) has the form $\phi(A)= \pm U A U^{*}$ for all $A \in \mathcal{B}(H)$, where $U$ is unitary. Also we show that a surjective map $\phi: \mathcal{B}(H) \rightarrow \mathcal{B}(K)$ satisfying

$$
W(\phi(B) \phi(A) \phi(B))=W(B A B) \quad \text { for all } A, B \in \mathcal{B}(H)
$$

Received by the editors May 1, 2004 and, in revised form, December 14, 2004.

2000 Mathematics Subject Classification. Primary 47B49; Secondary 47A12.

Key words and phrases. Hilbert spaces, numerical ranges, Jordan triple-products, Jordan isomorphisms.

This work was partially supported by NNSFC and PNSFS.

(C)2005 American Mathematical Society 
must be a multiple of a $\mathrm{C}^{*}$-isomorphism (by a cubic root of unity). In Section 3, we treat the problems for maps $\phi: \mathcal{S}^{a}(H) \rightarrow \mathcal{S}^{a}(K)$. In Section 4, we characterize the maps $\phi: \mathcal{B}(H) \rightarrow \mathcal{B}(K)$ satisfying

$$
W\left(\phi(A)^{*} \phi(B)\right)=W\left(A^{*} B\right) \text { for all } A, B \in \mathcal{B}(H),
$$

and

$$
W\left(\phi(B) \phi(A)^{*} \phi(B)\right)=W\left(B A^{*} B\right) \text { for all } A, B \in \mathcal{B}(H) .
$$

We obtain more general results covering these in the indefinite inner product space context. Some remarks and questions are given in Section 5.

\section{MAPS ON $\mathcal{B}(H)$}

In this section we discuss the question of characterizing maps which preserve numerical ranges of operator products or numerical ranges of operator Jordan tripleproducts. The following are our main results.

Theorem 2.1. Let $H$ and $K$ be complex Hilbert spaces and let $\phi: \mathcal{B}(H) \rightarrow \mathcal{B}(K)$ be a surjective map. Then $\phi$ satisfies $E q$. (1.1) if and only if there is a unitary operator $U: H \rightarrow K$ such that $\phi$ is of the form

$$
\phi(A)=\epsilon U A U^{*}
$$

for all $A \in \mathcal{B}(H)$, where $\epsilon= \pm 1$.

Theorem 2.2. Let $H$ and $K$ be complex Hilbert spaces and let $\phi: \mathcal{B}(H) \rightarrow \mathcal{B}(K)$ be a surjective map. Then $\phi$ satisfies Eq. (1.2) if and only if there is a scalar $\lambda$ with $\lambda^{3}=1$ and a unitary operator $U: H \rightarrow K$ such that either

(1) $\phi(A)=\lambda U A U^{*}$ for all $A \in \mathcal{B}(H)$; or

(2) $\phi(A)=\lambda U A^{t} U^{*}$ for all $A \in \mathcal{B}(H)$, where $A^{t}$ is the transpose of $A$ with respect to an arbitrarily fixed orthonormal basis of $H$.

The next lemma is crucial for our proofs of Theorem 2.1 and 2.2 as well as other results of this paper, which gives new characterizations of rank-one operators by numerical range of operator products.

Lemma 2.3. Let $A \in \mathcal{B}(H)$. The following conditions are equivalent:

(i) $A$ is a rank-one operator.

(ii) For every $B \in \mathcal{B}(H)$ with $A B \neq 0, W(A B)$ is either an ellipse which has 0 as a focus or a line segment which has 0 as an end point.

(iii) For every $B \in \mathcal{B}(H), B A B \neq 0$ implies that $W(B A B)$ is either an ellipse which has 0 as a focus or a line segment which has 0 as an end point.

Proof. (i) $\Rightarrow$ (ii) and (i) $\Rightarrow$ (iii) are obvious since, under the assumptions, $A B$ and $B A B$ are of rank one and the numerical range of every rank-one operator has the form stated in (ii).

(ii) $\Rightarrow($ i). Assume that $\operatorname{rank} A \geq 2$. Then there exist linearly independent vectors $x_{1}, x_{2} \in H$ such that $A x_{1} \perp A x_{2}$ and $\left\|A x_{1}\right\|=\left\|A x_{2}\right\|=1$. Let $B=\alpha x_{1} \otimes A x_{1}+$ $\beta x_{2} \otimes A x_{2}+\gamma x_{1} \otimes A x_{2}, \alpha, \beta, \gamma \in \mathbb{C}$. It follows that $A B=\alpha A x_{1} \otimes A x_{1}+\beta A x_{2} \otimes$ $A x_{2}+\gamma A x_{1} \otimes A x_{2}$. If $\alpha, \beta$ and $\gamma$ are all nonzero, then $W(A B)$ is an ellipse which has $\alpha, \beta$ as focuses, contradicting to the conditions of (ii).

(iii) $\Rightarrow$ (i). Assume, on the contrary, that $A$ satisfies (iii) but $\operatorname{rank} A \geq 2$. We have to show that there exists a $B \in \mathcal{B}(H)$ such that $W(B A B)$ is neither an ellipse with 0 as a focus, nor a line segment with 0 as an end point. 
If $\operatorname{dim} H \geq 4$, then there exists a rank-four projection $P$ such that $\operatorname{rank}(P A P) \geq$ 2. In fact, there exist vectors $x_{1}, x_{2}$ with $x_{1} \perp x_{2}$ such that $A x_{1}$ and $A x_{2}$ are linearly independent. Let $X=\left[x_{1}, x_{2}, A x_{1}, A x_{2}\right]$, the linear subspace spanned by $\left\{x_{1}, x_{2}, A x_{1}, A x_{2}\right\}$. Then $\operatorname{dim} X \leq 4$ and $P_{X} A P_{X}$ has rank $>1$. Here we denote by $P_{L}$ the projection with closed subspace $L$ as its range. Take any 4-dimensional subspace $H_{4}$ containing $X$ and let $P=P_{H_{4}}$. It is obvious that $\operatorname{rank}(P A P) \geq 2$.

Denote $A_{1}=\left.P A\right|_{H_{4}} \in \mathcal{B}\left(H_{4}\right)$. If we have shown that there is an operator $B_{1} \in \mathcal{B}\left(H_{4}\right)$ such that $B_{1} A_{1} B_{1} \neq 0,0 \in W\left(B_{1} A_{1} B_{1}\right)$ but $W\left(B_{1} A_{1} B_{1}\right)$ is neither an ellipse with 0 as a focus, nor a line segment with 0 as an end point, then, let $B=\left(\begin{array}{cc}B_{1} & 0 \\ 0 & 0\end{array}\right)$. It is clear that $W(B A B)$ is neither an ellipse with 0 as a focus, nor a line segment with 0 as an end point since $W(B A B)=\operatorname{conv}\left\{W\left(B_{1} A_{1} B_{1}\right) \cup\{0\}\right\}=$ $W\left(B_{1} A_{1} B_{1}\right)$ as $0 \in W\left(B_{1} A_{1} B_{1}\right)$. Where $\operatorname{conv}(\Lambda)$ denotes the convex hull of the set $\Lambda$. Thus we get a contradiction and then the proof of (iii) $\Rightarrow$ (i) for the case $\operatorname{dim} H \geq 4$ is completed. So, the task of proving (iii) $\Rightarrow$ (i) is reduced to the four dimensional case.

Identify $\mathcal{B}\left(H_{4}\right)$ with $M_{4}(\mathbb{C})$ and assume $A \in M_{4}(\mathbb{C})$ has rank greater than 1 . Then there exists a transformation $S: \mathbb{C}^{2} \rightarrow \mathbb{C}^{4}$ with $S^{*} S=I_{2}$ such that $S^{*} A S \in$ $M_{2}(\mathbb{C})$ is invertible. Thus there is a $2 \times 2$ unitary matrix $U=\left(\begin{array}{ll}u_{11} & u_{12} \\ u_{21} & u_{22}\end{array}\right)$ and there are positive numbers $s_{1}, s_{2}$ such that $S^{*} A S=\left(\begin{array}{cc}s_{1} & 0 \\ 0 & s_{2}\end{array}\right) U$. Take $T: \mathbb{C}^{2} \rightarrow \mathbb{C}^{4}$ so that $W=\left(\begin{array}{cc}S & T\end{array}\right) \in M_{4}(\mathbb{C})$ is unitary. Then

$$
W^{*} A W=\left(\begin{array}{cc}
S^{*} A S & S^{*} A T \\
T^{*} A S & T^{*} A T
\end{array}\right)=\left(\begin{array}{cc}
\left(\begin{array}{cc}
s_{1} & 0 \\
0 & s_{2}
\end{array}\right) U & S^{*} A T \\
T^{*} A S & T^{*} A T
\end{array}\right) .
$$

Pick nonzero complex numbers $b_{1}$ and $b_{2}$ so that $\bar{u}_{11} s_{1} b_{1}^{2}+\bar{u}_{22} s_{2} b_{2}^{2}=0$. Let

$$
\begin{aligned}
B=W & \left(U^{*}\left(\begin{array}{cc}
b_{1} & 0 \\
0 & b_{2}
\end{array}\right) \oplus 0\right) W^{*} \in M_{4}(\mathbb{C}) \text {. Then } \\
& W^{*} B A B W=U^{*}\left(\begin{array}{cc}
s_{1} b_{1}^{2} & 0 \\
0 & s_{2} b_{2}^{2}
\end{array}\right) \oplus 0=\left(\begin{array}{cc}
\bar{u}_{11} s_{1} b_{1}^{2} & \bar{u}_{21} s_{2} b_{2}^{2} \\
\bar{u}_{12} s_{1} b_{1}^{2} & \bar{u}_{22} s_{2} b_{2}^{2}
\end{array}\right) \oplus 0 .
\end{aligned}
$$

It is easily checked that the matrix $\left(\begin{array}{cc}\bar{u}_{11} s_{1} b_{1}^{2} & \bar{u}_{21} s_{2} b_{2}^{2} \\ \bar{u}_{12} s_{1} b_{1}^{2} & \bar{u}_{22} s_{2} b_{2}^{2}\end{array}\right)$ has two nonzero eigenvalues $\lambda_{1}$ and $\lambda_{2}$ with $\lambda_{1}+\lambda_{2}=0$, and hence its numerical range, as well as the numerical range of $B A B$, contains 0 but is neither an ellipse with 0 as a focus, nor a line segment with 0 as an end point. This completes the proof.

The rest of this section is devoted to proving Theorem 2.2. The proof of Theorem 2.1 is similar and we omit it here. To do this, we need two more lemmas which are also useful in Section 4.

For finite rank operators in $\mathcal{B}(H)$ one can define a trace functional tr by $\operatorname{tr}(A)=$ $\sum_{k=1}^{n}\left\langle x_{k}, f_{k}\right\rangle$ when $A=\sum_{k=1}^{n} x_{k} \otimes f_{k}$.

Lemma 2.4. Let $A, C \in \mathcal{B}(H)$. If $\operatorname{tr}(B A B)=\operatorname{tr}(B C B)$ for every rank-one projection $B \in \mathcal{B}(H)$, then $A=C$.

Proof. Let $B=x \otimes x$, where $x$ is a unit vector. Then $B$ is a rank-1 projection and every rank-1 projection takes this form. By the assumption, we have $\langle A x, x\rangle=$ 
$\operatorname{tr}(A x \otimes x)=\operatorname{tr}(B A B)=\operatorname{tr}(B C B)=\operatorname{tr}(C x \otimes x)=\langle C x, x\rangle$. Thus $\langle A x, x\rangle=\langle C x, x\rangle$ holds for every unit vector $x \in H$, which entails $A=C$ since $H$ is complex.

Lemma 2.5. Let $T \in \mathcal{B}(H)$ be invertible. Then $\left\langle T^{-1} x, f\right\rangle\langle T x, f\rangle=\langle x, f\rangle^{2}$ for every $x, f \in H$ implies that there exists a $\lambda \in \mathbb{C}$ such that $T=\lambda I$.

Proof. Fix a nonzero $x \in H$. Then, for every $f \in[x]^{\perp} \subset H$, we have $\left\langle T^{-1} x, f\right\rangle=0$ or $\langle T x, f\rangle=0$ since $\left\langle T^{-1} x, f\right\rangle\langle T x, f\rangle=\langle x, f\rangle^{2}=0$.

Let $M_{x}=\left\{f \in[x]^{\perp} \mid\langle T x, f\rangle=0\right\}$ and $N_{x}=\left\{f \in[x]^{\perp} \mid\left\langle T^{-1} x, f\right\rangle=0\right\}$. Then $M_{x} \cup N_{x}=[x]^{\perp}$. Because $[x]^{\perp}, M_{x}$ and $N_{x}$ are all closed linear subspaces, we must have $M_{x} \subseteq N_{x}=[x]^{\perp}$ or $N_{x} \subseteq M_{x}=[x]^{\perp}$.

If $N_{x}=[x]^{\perp}$, then $T^{-1} x \in[x]$. So there exists a $\lambda_{x} \in \mathbb{C}$ such that $T^{-1} x=$ $\lambda_{x} x \neq 0$, that is, $T x=\lambda_{x}{ }^{-1} x$.

If $M_{x}=[x]^{\perp}$, then $T x \in[x]$, that is, $T x=\lambda_{x} x$ for some scalar $\lambda_{x}$.

Since $x$ is arbitrary, we see that, for every $x \in H$, there is a scalar $\lambda_{x}$ such that $T x=\lambda_{x} x$. This implies that there exists a $\lambda \in \mathbb{C}$ such that $T=\lambda I$.

Now we are in a position to prove Theorem 2.2. Note that, if two rank-one operators have the same numerical ranges, then they have the same nonzero eigenvalues, and hence have the same traces. This simple observation will be used frequently in this paper.

Proof of Theorem 2.2. It is clear that we need only to check the necessity. Suppose that $\phi$ satisfies Eq. (1.2). For the sake of simplicity we assume $K=H$.

First we check that $\phi$ preserves rank-one operators in both directions. Let $A \in$ $\mathcal{B}(H)$ be a rank-one operator. For every $T \in \mathcal{B}(H)$, there exists a $B \in \mathcal{B}(H)$ such that $T=\phi(B)$. It follows from $W(T \phi(A) T)=W(B A B)$ and Lemma 2.3 that $\phi(A)$ is a rank-one operator. Similarly, $\phi(A)$ is a rank-one operator will imply that $A$ is a rank-one operator, too.

Second we show that $\phi$ is linear. Let $A, A^{\prime} \in \mathcal{B}(H)$ be arbitrarily given and let $B \in \mathcal{B}(H)$ be a rank-one operator. Notice that, for rank-one operators $T$ and $S$, $W(T)=W(S)$ will imply $\operatorname{tr}(T)=\operatorname{tr}(S)$. Then Eq. (1.2) implies that

$$
\begin{gathered}
\operatorname{tr}\left(\phi(B)\left(\phi\left(A+A^{\prime}\right) \phi(B)\right)=\operatorname{tr}\left(B\left(A+A^{\prime}\right) B\right)=\operatorname{tr}(B A B)+\operatorname{tr}\left(B A^{\prime} B\right)\right. \\
=\operatorname{tr}(\phi(B) \phi(A) \phi(B))+\operatorname{tr}\left(\phi(B) \phi\left(A^{\prime}\right) \phi(B)\right)=\operatorname{tr}\left(\phi(B)\left(\phi(A)+\phi\left(A^{\prime}\right)\right) \phi(B)\right) .
\end{gathered}
$$

Since $\phi(B)$ runs over all rank-one operators when $B$ runs over all rank-one operators, Lemma 2.4 ensures that $\phi\left(A+A^{\prime}\right)=\phi(A)+\phi\left(A^{\prime}\right)$, i.e., $\phi$ is additive. Similarly, we can check that $\phi$ is homogeneous.

So, $\phi$ is a linear bijection on $\mathcal{B}(H)$ preserving rank-one operators in both directions. It follows from [8, Lemma 1.2], either

(i) there exist bijective linear operators $U$ and $V$ on $H$ such that $\phi(x \otimes f)=$ $U x \otimes V f(\forall x, f \in H)$; or

(ii) there exist bijective conjugate linear operators $U$ and $V$ on $H$ such that $\phi(x \otimes f)=U f \otimes V x(\forall x, f \in H)$.

Suppose the case (i) occurs, we will show that $\phi$ has the form (1) stated in Theorem 2.2. By Eq. (1.2), we have $W(x \otimes f)=W(\phi(I) \phi(x \otimes f) \phi(I))$. So, by taking trace, $\langle x, f\rangle=\left\langle\phi(I)^{2} U x, V f\right\rangle$ holds for every $x, f \in H$. It follows that $U, V$ are bounded and $V^{*} \phi(I)^{2} U=I$. Eq. (1.2) also yields that $\phi(I)^{2}=$ $\phi(I)^{-1}$. Hence $V^{*} \phi(I)^{-1} U=I$, i.e., $\phi(I)=U V^{*}$. As $V^{*} \phi(I) U=V^{*} U V^{*} U$ and 
$V^{*} U V^{*} U V^{*} U=I$, we have $V^{*} U V^{*} U=\left(V^{*} U\right)^{-1}$. Since $W((x \otimes f) I(x \otimes f))=$ $W((U x \otimes V f) \phi(I)(U x \otimes V f))$, we see that $\langle x, f\rangle^{2}=\left\langle V^{*} \phi(I) U x, f\right\rangle\left\langle V^{*} U x, f\right\rangle$, that is, $\langle x, f\rangle^{2}=\left\langle\left(V^{*} U\right)^{-1} x, f\right\rangle\left\langle V^{*} U x, f\right\rangle$ holds for all $x, f \in H$. By Lemma 2.5, there exists a $\lambda \in \mathbb{C}$ such that $V^{*} U=\lambda I$. Notice that $I=\left(V^{*} U\right)^{3}=\lambda^{3} I$, so $\lambda^{3}=1$ and $V^{*}=\lambda U^{-1}$. Thus we have $\phi(x \otimes f)=\lambda U(x \otimes f) U^{-1}$ for all rank-one operators $x \otimes f$, where $\lambda^{3}=1$.

Now, for every $A$, we have

$$
W\left(\lambda^{2} U(x \otimes f) U^{-1} \phi(A) U(x \otimes f) U^{-1}\right)=W((x \otimes f) A(x \otimes f)) .
$$

So $\operatorname{tr}\left(\lambda^{2}(x \otimes f) U^{-1} \phi(A) U(x \otimes f)\right)=\operatorname{tr}((x \otimes f) A(x \otimes f))$. It follows from Lemma 2.4 again that $\lambda^{2} U^{-1} \phi(A) U=A$. Hence $\phi(A)=\lambda U A U^{-1}$ holds for every $A \in \mathcal{B}(H)$.

Let $x \in H$ be a unit vector; then we have

$$
\begin{aligned}
{[0,1] } & =W((x \otimes x)(x \otimes x)(x \otimes x))=W(\phi(x \otimes x) \phi(x \otimes x) \phi(x \otimes x)) \\
& =W\left(U(x \otimes x) U^{-1} U(x \otimes x) U^{-1} U(x \otimes x) U^{-1}\right)=W\left(U x \otimes x U^{-1}\right) .
\end{aligned}
$$

This implies that $U x \otimes\left(U^{-1}\right)^{*} x$ is a rank-one projection and hence $\left(U^{-1}\right)^{*} x$ is linearly dependent of $U x$ for every unit vector $x$. It follows that $\left(U^{-1}\right)^{*} \in[U]$ and there exists a $\mu>0$ such that $U U^{*}=\mu I$. Let $U_{1}=(\sqrt{\mu})^{-1} U$, then $U_{1} U_{1}{ }^{*}=I$. So $\phi(A)=\lambda U_{1} A U_{1}{ }^{*}$ for all $A \in \mathcal{B}(H)$, where $\lambda^{3}=1$ and $U_{1}$ is unitary. Hence $\phi$ has the form (1) stated in Theorem 2.2.

Assume the case (ii) occurs, let us show that the form (2) in Theorem 2.2 holds true. Taking $A=x \otimes f$ and $B=I$ in the equation (1.2), we get

$$
W(\phi(I)(U f \otimes V x) \phi(I))=W(\phi(I) \phi(x \otimes f) \phi(I))=W(x \otimes f) .
$$

Note that both $U$ and $V$ are conjugate linear. So by taking trace we have $\langle x, f\rangle=$ $\left\langle\phi(I) U f, \phi(I)^{*} V x\right\rangle=\left\langle x, V^{*} \phi(I)^{2} U f\right\rangle$ for every $x, f \in H$. Now it is easily checked that both $U$ and $V$ are bounded, and $V^{*} \phi(I)^{2} U=I$. Thus, similar to the corresponding part in the proof of case (i) above, one gets $\phi(I)^{2}=\phi(I)^{-1}, \phi(I)=U V^{*}$, and $V^{*} U V^{*} U=\left(V^{*} U\right)^{-1}$. The equation

$$
W((x \otimes f) I(x \otimes f))=W((U f \otimes V x) \phi(I)(U f \otimes V x))
$$

yields that

$$
\begin{aligned}
\langle x, f\rangle^{2} & =\langle\phi(I) U f, V x\rangle\langle U f, V x\rangle=\left\langle x, V^{*} \Phi(I) U f\right\rangle\left\langle x, V^{*} U f\right\rangle \\
& =\left\langle x,\left(V^{*} U\right)^{-1} f\right\rangle\left\langle x, V^{*} U f\right\rangle
\end{aligned}
$$

for every $x, f$. By Lemma 2.5 we see that $V^{*} U=\lambda I$ with $\lambda^{3}=1$. Now for every $A$, it follows from $W((U f \otimes V x) \phi(A)(U f \otimes V x))=W((x \otimes f) A(x \otimes f))$ that

$$
\begin{aligned}
\operatorname{tr}((x \otimes & f) A(x \otimes f)) \\
& =\operatorname{tr}((U f \otimes V x) \phi(A)(U f \otimes V x))=\langle\phi(A) U f, V x\rangle\langle U f, V x\rangle \\
& =\left\langle U^{*} \phi(A)^{*} V x, f\right\rangle\left\langle x, V^{*} U f\right\rangle=\left\langle U^{*} \phi(A)^{*}\left(\bar{\lambda}\left(U^{*}\right)^{-1}\right) x, f\right\rangle\langle x, \lambda f\rangle \\
& =\left\langle\lambda^{2} U^{*} \phi(A)^{*}\left(U^{*}\right)^{-1} x, f\right\rangle\langle x, f\rangle \\
& =\operatorname{tr}\left((x \otimes f)\left(\lambda^{2} U^{*} \phi(A)^{*}\left(U^{*}\right)^{-1}\right)(x \otimes f)\right)
\end{aligned}
$$

for every rank-one operator $x \otimes f$. By Lemma 2.4, we obtain that

$$
\lambda^{2} U^{*} \phi(A)^{*}\left(U^{*}\right)^{-1}=A,
$$

that is,

$$
\phi(A)=\bar{\lambda} U A^{*} U^{-1}
$$


for every $A \in \mathcal{B}(H)$. Now it is trivial to check that $U$ can be taken as a conjugate unitary operator. Pick an orthonormal basis $\left\{e_{j} \mid j \in \mathcal{J}\right\}$ and define a conjugate unitary operator $J: H \rightarrow H$ by $J x=\sum_{j \in \mathcal{J}} \overline{\xi_{j}} e_{j}$ if $x=\sum_{j \in \mathcal{J}} \xi_{j} e_{j}$. It is clear that $J^{2}=I, J^{*}=J$ and $A^{*}=J A^{t} J$, where $A^{t}$ is the transpose of $A$ with respect to the basis $\left\{e_{j} \mid j \in \mathcal{J}\right\}$. Let $U_{1}=U J$ and $\alpha=\bar{\lambda}$. Then, $U_{1}$ is a unitary operator, $\alpha^{3}=1$ and $\phi(A)=\alpha U_{1} A^{t} U_{1}^{*}$ for all $A \in \mathcal{B}(H)$, finishing the proof.

\section{MAPS ON THE SPACE OF SELF-ADJOINT OPERATORS}

In this section we characterize the maps on the real linear subspace $\mathcal{S}^{a}(H)$ of all self-adjoint operators on a complex Hilbert space which preserve the numerical ranges of products of operators.

Theorem 3.1. Let $H, K$ be complex Hilbert spaces and let $\phi: \mathcal{S}^{a}(H) \rightarrow \mathcal{S}^{a}(K)$ be a surjective map. Then

$$
W(\phi(A) \phi(B))=W(A B)
$$

for all $A, B \in \mathcal{S}^{a}(H)$ if and only if there is a unitary operator $U: H \rightarrow K$ such that $\phi$ is of the form

$$
\phi(A)=\epsilon U A U^{*}
$$

for all $A \in \mathcal{S}^{a}(H)$, where $\epsilon= \pm 1$.

Similar to Section 2, our proofs are based on the following characterizations of rank-one self-adjoint operators.

Lemma 3.2. Let $A \in \mathcal{S}^{a}(H)$. The following conditions are equivalent:

(i) $A$ is of rank one.

(ii) For every $B \in \mathcal{S}^{a}(H), A B \neq 0$ implies that $W(A B)$ is either an ellipse with 0 as a focus or a line segment with 0 as an end point.

Proof of Theorem 3.1. Suppose that $\Phi$ satisfies Eq. (3.1). Applying Lemma 3.2 we can prove that $\phi$ preserves rank-one operators in both directions and $\phi$ is real linear. Thus $\phi$ preserves adjacency in both directions with $\phi(0)=0$. By [7], there exists a bijective linear or conjugate linear operator $V$ on $H$ and a real scalar $c \in \mathbb{R} \backslash\{0\}$ such that $\phi(x \otimes x)=c V x \otimes V x$ for all $x \in H$.

For every $x \in H$ with $\|x\|=1$, we have $x \otimes x=(x \otimes x)(x \otimes x)$. Hence

$$
[0,1]=W(x \otimes x)=W\left(\phi(x \otimes x)^{2}\right)=c^{2}\|V x\|^{2}\left[0,\|V x\|^{2}\right] .
$$

It follows that $c^{2}\|V x\|^{4}=1,\|V x\|=\frac{1}{\sqrt{|c|}}\|x\|$ for every $x \in H$. Let $U=\sqrt{|c|} V$; then $U$ is a unitary or conjugate unitary operator such that

$$
\phi(x \otimes x)=c V x \otimes V x=c \frac{1}{\sqrt{|c|}} U x \otimes \frac{1}{\sqrt{|c|}} U x=c|c|^{-1} U x \otimes U x=\epsilon U x \otimes U x
$$

with $\epsilon= \pm 1$.

There is no loss of generality in assuming that $\epsilon=1$.

If $U$ is unitary, then for every rank-one operator $T \in \mathcal{S}^{a}(H)$, we have $\phi(T)=$ $U T U^{*}$. Thus, for each $A \in \mathcal{S}^{a}(H)$,

$$
\begin{aligned}
W(A x \otimes x) & =W(\phi(A) \phi(x \otimes x))=W\left(\phi(A) U(x \otimes x) U^{*}\right) \\
& =W\left(U^{*} \phi(A) U x \otimes x\right)
\end{aligned}
$$

and hence

$$
\left\langle U^{*} \phi(A) U x, x\right\rangle=\langle A x, x\rangle
$$


for all $x \in H$. This ensures that

$$
\phi(A)=U A U^{*} \quad \text { for all } A \in \mathcal{S}^{a}(H),
$$

that is, $\phi$ has the form stated in the theorem.

We assert the case that $U$ is a conjugate unitary operator cannot occur. Assume on the contrary that $U$ is conjugate unitary such that $\phi(x \otimes x)=U x \otimes U x$ for all $x \in H$. It follows that, for every $A \in \mathcal{S}^{a}(H)$ and every $x \in H$,

$$
W(A x \otimes x)=W(\phi(A) \phi(x \otimes x))=W(\phi(A) U x \otimes U x)
$$

and consequently,

$$
\langle x, A x\rangle=\langle A x, x\rangle=\langle\phi(A) U x, U x\rangle=\left\langle x, U^{*} \phi(A) U x\right\rangle .
$$

Thus we still have $\phi(A)=U A U^{*}$ for every $A \in \mathcal{S}^{a}(H)$. On the other hand, for $T \in \mathcal{B}(H)$,

$$
\left\langle U T U^{*} x, x\right\rangle=\left\langle U^{*} x, T U^{*} x\right\rangle=\left\langle T^{*} U^{*} x, U^{*} x\right\rangle,
$$

so $W\left(U T U^{*}\right)=W\left(T^{*}\right)=W(T)^{*}$. Thus we get

$$
W(A B)=W(\phi(A) \phi(B))=W\left(U A B U^{*}\right)=W(A B)^{*}=W(B A)
$$

for all $A, B \in \mathcal{S}^{a}(H)$, which is impossible. The proof is completed.

\section{MAPs PRESERVING NUMERICAL RANGES OF SKEW PRODUCTS}

The purpose of this section is to classify the maps which preserve numerical ranges of skew products or Jordan skew triple-products of operators on Hilbert spaces, i.e., the maps $\phi$ which satisfy

$$
W\left(\phi(A)^{*} \phi(B)\right)=W\left(A^{*} B\right) \quad \text { or } \quad W\left(\phi(B) \phi(A)^{*} \phi(B)\right)=W\left(B A^{*} B\right) .
$$

Taking indefinite inner product structures into consideration, we discuss it here in a more general situation. In fact, we show that

Theorem 4.1. Let $H_{i}$ be complex Hilbert spaces and $S_{i} \in \mathcal{B}\left(H_{i}\right)$ invertible selfadjoint operators, $i=1,2$. Let $\phi: \mathcal{B}\left(H_{1}\right) \rightarrow \mathcal{B}\left(H_{2}\right)$ be a surjective map. Then

$$
W\left(S_{2}^{-1} \phi(A)^{*} S_{2} \phi(B)\right)=W\left(S_{1}^{-1} A^{*} S_{1} B\right)
$$

holds for all $A, B \in \mathcal{B}\left(H_{1}\right)$ if and only if there exist a nonzero real number $c \in$ $\mathbb{R} \backslash\{0\}$, a linear invertible bounded operator $U \in \mathcal{B}\left(H_{1}, H_{2}\right)$ and a unitary operator $V \in \mathcal{B}\left(H_{1}, H_{2}\right)$ satisfying $U^{*} S_{2} U=c U S_{1}$ and $S_{2} V=c V S_{1}$, respectively, such that

$$
\phi(A)=U A V^{*}
$$

for all $A \in \mathcal{B}\left(H_{1}\right)$.

Theorem 4.2. Let $H_{i}$ be complex Hilbert spaces and $S_{i} \in \mathcal{B}\left(H_{i}\right)$ invertible selfadjoint operators, $i=1,2$. Let $\phi: \mathcal{B}\left(H_{1}\right) \rightarrow \mathcal{B}\left(H_{2}\right)$ be a surjective map. Then

$$
W\left(\phi(B) S_{2}^{-1} \phi(A)^{*} S_{2} \phi(B)\right)=W\left(B S_{1}{ }^{-1} A^{*} S_{1} B\right)
$$

for all $A, B \in \mathcal{B}\left(H_{1}\right)$ if and only if there exist a number $c \in \mathbb{R} \backslash\{0\}$ and a unitary operator $U$ such that either

(1) $U^{*} S_{2}^{-1} U S_{1}=c I$ and $\phi(A)=U A U^{*}$ for all $A \in \mathcal{B}\left(H_{1}\right)$; or

(2) $S_{1}^{t} U^{*} S_{2} U=c I$ and $\phi(A)=U A^{t} U^{*}$ for all $A \in \mathcal{B}\left(H_{1}\right)$. Where $A^{t}$ is the transpose of $A$ with respect to an arbitrarily fixed basis. 
In particular, if both $S_{1}$ and $S_{2}$ are the identity, we have

Corollary 4.3. Let $H, K$ be complex Hilbert spaces and let $\phi: \mathcal{B}(H) \rightarrow \mathcal{B}(K)$ be a surjective map. Then

$$
W\left(\phi(A)^{*} \phi(B)\right)=W\left(A^{*} B\right)
$$

for all $A, B \in \mathcal{B}(H)$ if and only if there exist unitary operators $U$ and $V$ in $\mathcal{B}\left(H_{1}, H_{2}\right)$ such that $\phi$ is of the form

$$
\phi(A)=U A V^{*}
$$

for all $A \in \mathcal{B}(H)$.

Corollary 4.4. Let $H, K$ be complex Hilbert spaces and let $\phi$ be a surjective map from $\mathcal{B}(H)$ onto $\mathcal{B}(K)$. Then

$$
W\left(\phi(B) \phi(A)^{*} \phi(B)\right)=W\left(B A^{*} B\right)
$$

for all $A, B \in \mathcal{B}(H)$ if and only if there exists a unitary operator $U$ such that either $\phi(A)=U A U^{*}$ for all $A$, or $\phi(A)=U A^{t} U^{*}$ for all $A$.

So, Eq. (4.3) and Eq. (4.4) give a characterization of $*$-isomorphisms multiplied by a unitary and a characterization of $\mathrm{C}^{*}$-isomorphisms between $\mathcal{B}(H)$ and $\mathcal{B}(K)$, respectively.

We give a proof of Theorem 4.2 here. The proof of Theorem 4.1 is similar.

Proof of Theorem 4.2. It is clear that we need only check the "only if" part. Assume that $\phi$ satisfies the Eq. (4.2). For $A \in \mathcal{B}\left(H_{i}\right), T \in \mathcal{B}\left(H_{1}, H_{2}\right)$ and $S \in$ $\mathcal{B}\left(H_{2}, H_{1}\right)$, we denote $A^{\dagger}=S_{i}^{-1} A^{*} S_{i}, T^{\dagger}=S_{1}^{-1} T^{*} S_{2}$ and $S^{\dagger}=S_{2}^{-1} S^{*} S_{1}$, respectively. It is clear that $\dagger$ is an involution, i.e., $\left(B^{\dagger}\right)^{\dagger}=B,(B C)^{\dagger}=C^{\dagger} B^{\dagger}$, $(\alpha B+C)^{\dagger}=\bar{\alpha} B^{\dagger}+C^{\dagger}$ and $\left(B^{-1}\right)^{\dagger}=\left(B^{\dagger}\right)^{-1}$. Thus the equation (4.2) becomes

$$
W\left(\phi(B) \phi(A)^{\dagger} \phi(B)\right)=W\left(B A^{\dagger} B\right)
$$

for every $A, B \in \mathcal{B}\left(H_{1}\right)$ and $\phi$ satisfies Eq. (4.5).

Similar to the proof of Theorem 2.2 in Section 2, we can show that $\phi$ is a linear bijection preserving rank-one operators in both directions. Thus, either

(i) there exist bijective linear operators $U: H_{1} \rightarrow H_{2}$ and $V: H_{2} \rightarrow H_{1}$ such that $\phi(x \otimes f)=U x \otimes V f\left(\forall x, f \in H_{1}\right)$; or

(ii) there exist bijective conjugate linear operators $U: H_{1} \rightarrow H_{2}$ and $V: H_{2} \rightarrow$ $H_{1}$ such that $\phi(x \otimes f)=U f \otimes V x\left(\forall x, f \in H_{1}\right)$.

Suppose first that $\phi$ takes the form (i). Let $A=B=I$ in (4.5), we get $\phi(I) \phi(I)^{\dagger} \phi(I)=I$. So $\phi(I)$ is invertible and $\phi(I)^{\dagger}=\phi(I)^{-2}$.

Let $B=I$ and $A=x \otimes f$ in (4.5), we get

$$
\begin{aligned}
& W\left(\phi(I) S_{2}{ }^{-1}(V f \otimes U x) S_{2} \phi(I)\right)=W\left(\phi(I) \phi(x \otimes f)^{\dagger} \phi(I)\right) \\
= & W\left((x \otimes f)^{\dagger}\right)=W\left(S_{1}^{-1}(f \otimes x) S_{1}\right) .
\end{aligned}
$$

So $\left\langle\phi(I) S_{2}{ }^{-1} V f, \phi(I)^{*} S_{2} U x\right\rangle=\left\langle S_{1}{ }^{-1} f, S_{1} x\right\rangle=\langle f, x\rangle$. It follows that $U$ and $V$ are bounded, and $\left(\phi(I)^{*} S_{2} U\right)^{*} \phi(I) S_{2}{ }^{-1} V=I$, i.e.,

$$
U^{*} S_{2} \phi(I)^{2} S_{2}{ }^{-1} V=I .
$$

As $\phi(I)^{\dagger}=\phi(I)^{-2}$, we have $\phi(I)=U V^{*}$, and consequently,

$$
\phi(I)^{\dagger}=\phi(I)^{-2}=\left(V^{*}\right)^{-1} U^{-1}\left(V^{*}\right)^{-1} U^{-1} .
$$


Taking $A=I$ and $B=x \otimes f\left(x, f \in H_{1}\right)$ in Eq. (4.5), we get

$W\left((U x \otimes V f) \phi(I)^{\dagger}(U x \otimes V f)\right)=W\left(\phi(x \otimes f) \phi(I)^{\dagger} \phi(x \otimes f)\right)=W((x \otimes f)(x \otimes f))$.

This yields

$$
\left\langle\phi(I)^{\dagger} U x, V f\right\rangle\langle U x, V f\rangle=\langle x, f\rangle^{2}
$$

for every $x, f \in H_{1}$. So $\left\langle V^{*} \phi(I)^{\dagger} U x, f\right\rangle\left\langle V^{*} U x, f\right\rangle=\langle x, f\rangle^{2}$, that is,

$$
\left\langle U^{-1}\left(V^{*}\right)^{-1} x, f\right\rangle\left\langle V^{*} U x, f\right\rangle=\langle x, f\rangle^{2}
$$

for every $x, f \in H_{1}$. By Lemma 2.5 in Section 2 we see that there exists a $\lambda \in \mathbb{C}$ such that $V^{*} U=\lambda I$. Hence $V^{*}=\lambda U^{-1}, \phi(I)=U V^{*}=\lambda I$ and $\bar{\lambda} I=\phi(I)^{\dagger}=$ $\phi(I)^{-2}=\lambda^{-2} I$. Since $|\lambda|^{2} \lambda=1$, we get $\lambda=1$. It follows that

$$
\phi(x \otimes f)=U(x \otimes f) U^{-1}
$$

for every $x \otimes f \in \mathcal{B}\left(H_{1}\right)$.

Now, let $A \in \mathcal{B}\left(H_{1}\right)$. For any rank-one operator $B=x \otimes f$, Eq. (4.5) gives that

$$
\begin{aligned}
& W\left(U(x \otimes f) U^{-1} \phi(A)^{\dagger} U(x \otimes f) U^{-1}\right) \\
= & W\left(\phi(x \otimes f) \phi(A)^{\dagger} \phi(x \otimes f)\right)=W\left((x \otimes f) A^{\dagger}(x \otimes f)\right) .
\end{aligned}
$$

Thus we have $\operatorname{tr}\left((x \otimes f) U^{-1} \phi(A)^{\dagger} U(x \otimes f)\right)=\operatorname{tr}\left((x \otimes f) A^{\dagger}(x \otimes f)\right)$ for every $x \otimes f$, and by Lemma 2.4 in Section 2, we get $U^{-1} \phi(A)^{\dagger} U=A^{\dagger}$. So we have shown that

$$
\phi(A)=\left(U^{-1}\right)^{\dagger} A U^{\dagger}=\left(U^{\dagger}\right)^{-1} A U^{\dagger}
$$

holds for every $A \in \mathcal{B}\left(H_{1}\right)$. Eqs. (4.6) and (4.7) together yield that

$$
\phi(x \otimes f)=\left(U^{-1}\right)^{\dagger}(x \otimes f) U^{\dagger}=U(x \otimes f) U^{-1}
$$

for every $x, f \in H_{1}$. So $\left(U^{-1}\right)^{\dagger} x$ and $U x$ are linearly dependent for every $x \in H_{1}$, and then, there exists a $\mu \in \mathbb{R} \backslash\{0\}$ such that $U U^{\dagger}=\mu I$.

In Eq. (4.5), let $A=I$ and $B=x \otimes x$, where $x \in H_{1}$ with $\|x\|=1$. Then $[0,1]=W(x \otimes x)=W\left(\left(U^{\dagger}\right)^{-1}(x \otimes x) U^{\dagger}\right)$, this entails that $\left(U^{\dagger}\right)^{-1} x$ and $\left(U^{\dagger}\right)^{*} x$ are linearly dependent. So there exists a scalar $\alpha>0$ such that $\left(U^{\dagger}\right)^{*} U^{\dagger}=\alpha I$. This implies that there is a unitary operator $U_{1}$ and an $\alpha_{0} \in \mathbb{R}$ such that $\alpha_{0}^{2}=\alpha$ and $\left(U^{\dagger}\right)^{*}=\alpha_{0} U_{1}$. Thus we have

$$
\phi(A)=U_{1} A U_{1}^{*}
$$

for every $A \in \mathcal{B}\left(H_{1}\right)$. Note that $\mu I=U U^{\dagger}=\alpha_{0}{ }^{2}\left(U_{1}{ }^{*}\right)^{\dagger} U_{1}{ }^{*}=\alpha\left(U_{1}{ }^{*}\right)^{\dagger} U_{1}{ }^{*}$. Let $c=\alpha \mu^{-1}$; then $U_{1}^{\dagger} U_{1}=c I$, i.e.,

$$
S_{1}^{-1} U_{1}^{*} S_{2} U_{1}=c I \text {. }
$$

So $\phi$ has the form stated in Theorem 4.2 (1).

Assume that the case (ii) occurs. We will show that $\phi$ has the form stated in Theorem 4.2(2). Note that $U$ and $V$ are conjugate linear bijections, a similar argument as that in the beginning of case (i) shows that both $U$ and $V$ are bounded, $\phi(I)^{\dagger}=\phi(I)^{-2}$ and $\phi(I)=U V^{*}$. Let $A=I$ and $B=x \otimes f$ in Eq. (4.5), then we get

$W\left((U f \otimes V x) \phi(I)^{\dagger}(U f \otimes V x)\right)=W\left(\phi(x \otimes f) \phi(I)^{\dagger} \phi(x \otimes f)\right)=W((x \otimes f)(x \otimes f))$.

This implies that

$$
\left\langle\phi(I)^{\dagger} U f, V x\right\rangle\langle U f, V x\rangle=\langle x, f\rangle^{2}
$$

for every $x, f \in H_{1}$. So $\left\langle x, V^{*} \phi(I)^{\dagger} U f\right\rangle\left\langle x, V^{*} U f\right\rangle=\langle x, f\rangle^{2}$, that is,

$$
\left\langle x, U^{-1}\left(V^{*}\right)^{-1} f\right\rangle\left\langle x, V^{*} U f\right\rangle=\langle x, f\rangle^{2}
$$


for every $x, f \in H_{1}$. By Lemma 2.5 we see that there exists a $\lambda \in \mathbb{C}$ such that $V^{*} U=\lambda I$. Hence $V^{*}=\lambda U^{-1}, \phi(I)=U V^{*}=\lambda I$ and $\bar{\lambda} I=\phi(I)^{\dagger}=\phi(I)^{-2}=$ $\lambda^{-2} I$. It follows that $\lambda=1$ and

$$
\phi(x \otimes f)=U f \otimes\left(U^{*}\right)^{-1} x
$$

for every $x \otimes f \in \mathcal{B}\left(H_{1}\right)$. Let $A \in \mathcal{B}\left(H_{1}\right)$. For any rank-one operator $x \otimes f$, Eqs. (4.5) and (4.8) together imply that

$$
W\left(\left(U f \otimes\left(U^{*}\right)^{-1} x\right) \phi(A)^{\dagger}\left(U f \otimes\left(U^{*}\right)^{-1} x\right)\right)=W\left((x \otimes f) A^{\dagger}(x \otimes f)\right),
$$

and hence

$$
\operatorname{tr}\left((x \otimes f) U^{*}\left(\phi(A)^{\dagger}\right)^{*}\left(U^{*}\right)^{-1}(x \otimes f)\right)=\operatorname{tr}\left((x \otimes f) A^{\dagger}(x \otimes f)\right) .
$$

Applying Lemma 2.4, we see that $U^{*}\left(\phi(A)^{\dagger}\right)^{*}\left(U^{*}\right)^{-1}=A^{\dagger}$, i.e.,

$$
U^{*} S_{2} \phi(A) S_{2}^{-1}\left(U^{*}\right)^{-1}=S_{1}^{-1} A^{*} S_{1} .
$$

Thus we have proved that

$$
\phi(A)=U_{1} A^{*} U_{1}^{-1}
$$

for every $A \in \mathcal{B}\left(H_{1}\right)$, where $U_{1}=\left(S_{1} U^{*} S_{2}\right)^{-1}$ is a conjugate linear operator. Then, for any $x \otimes f$ we have

$$
\left(U_{1} f \otimes x\right) U_{1}^{-1}=\phi(x \otimes f)=U f \otimes\left(U^{*}\right)^{-1} x,
$$

this implies that $U_{1} f$ is linearly dependent of $U f$ for every $f \in H_{1}$. Thus we must have $U_{1}=\alpha U$ and $\phi(x \otimes f)=U_{1} f \otimes\left(U_{1}^{*}\right)^{-1} x$ for every $x \otimes f$.

Now, for every unit vector $x \in H_{1}$, since

$$
\begin{aligned}
{[0,1] } & =W(x \otimes x)=W\left(\phi(x \otimes x) \phi(I)^{\dagger} \phi(x \otimes x)\right) \\
& =W\left(U_{1}(x \otimes x) U_{1}^{-1} U_{1}(x \otimes x) U_{1}^{-1}\right)=W\left(U_{1} x \otimes\left(U_{1}^{*}\right)^{-1} x\right),
\end{aligned}
$$

we see that $\left(U_{1}^{*}\right)^{-1}=\beta U_{1}$ for some $\beta>0$. Let $V_{1}=\sqrt{\beta} U_{1}$. Then $V_{1}$ is conjugate unitary and

$$
\phi(A)=V_{1} A^{*} V_{1}^{*}
$$

for all $A \in \mathcal{B}\left(H_{1}\right)$. Equivalently, there exists a unitary operator $U_{2}: H_{1} \rightarrow H_{2}$ such that

$$
\phi(A)=U_{2} A^{t} U_{2}^{*}
$$

for all $A$, where $A^{t}$ is the transpose of $A$ with respect to an arbitrarily fixed orthonormal basis. By substituting Eq. (4.10) in Eq. (4.2) with $B$ being rank one, and noting that $W\left(T^{t}\right)=W(T)$, it is easily checked that

$$
\operatorname{tr}\left(B^{t} U_{2}^{*} S_{2}^{-1} U_{2}\left(A^{t}\right)^{*} U_{2}^{*} S_{2} U_{2} B^{t}\right)=\operatorname{tr}\left(B^{t}\left(S_{1}^{-1} A^{*} S_{1}\right)^{t} B^{t}\right) .
$$

By Lemma 2.4 again, we get $U_{2}^{*} S_{2}^{-1} U_{2}\left(A^{t}\right)^{*} U_{2}^{*} S_{2} U_{2}=\left(S_{1}^{-1} A^{*} S_{1}\right)^{t}$. This implies that there is a scalar $c$ such that $U_{2}^{*} S_{2} U_{2}=c\left(S_{1}^{t}\right)^{-1}$. So $S_{1}^{t} U_{2}^{*} S_{2} U_{2}=c I$ as desired. The proof is finished. 


\section{Remarks AND QUESTIONS}

Before concluding this paper we give some remarks and propose a question.

Remark 5.1. The results in this paper are still valid if we replace the numerical range by the closure of numerical range. Moreover, Theorems 2.1 and 2.2 are still true if we replace $\mathcal{B}(H)$ and $\mathcal{B}(K)$ by standard operator algebras $\mathcal{A}$ on $H$ and $\mathcal{B}$ on $K$, respectively. Recall that a standard operator algebra on $H$ is a subalgebra of $\mathcal{B}(H)$ which contains the identity $I$ and all finite rank operators. Similarly, Theorems 4.1 and 4.2 still hold if we replace $\mathcal{B}\left(H_{i}\right)(\mathcal{B}(H)$ and $\mathcal{B}(K))$ by standard $\dagger$-operator algebras $\mathcal{A}_{i}$ on $H_{i}(\mathcal{A}$ on $H$ and $\mathcal{B}$ on $K$, resp.).

Remark 5.2. Based on the results and the methods in this paper, it is not difficult to classify the surjective maps that preserve numerical ranges of $k$-products (or skew $k$-products) of operators with $k>2$ of the form $A_{1}^{\tau_{1}} A_{2}^{\tau_{2}} \cdots A_{k}^{\tau_{k}}$, where $A_{i}^{\tau_{i}}=A_{i}$ or $A_{i}^{*}$. For example, let $\phi: \mathcal{B}(H) \rightarrow \mathcal{B}(K)$ be a surjection such that

$$
W\left(\phi\left(A_{1}\right) \phi\left(A_{2}\right) \ldots \phi\left(A_{k}\right)\right)=W\left(A_{1} A_{2} \ldots A_{k}\right)
$$

for all $A_{1}, A_{2}, \ldots, A_{k} \in \mathcal{B}(H)$. Then particularly we have $W\left(\phi(I)^{k-2} \phi(A) \phi(B)\right)=$ $W\left(\phi(A) \phi(B) \phi(I)^{k-2}\right)=W(A B)$ for all $A, B$. Similar to the proof of Theorem 2.2 , it is easily checked that $\phi(I)$ is invertible, $\phi$ is linear and preserves rank-one operators in both directions. It follows that $\phi(I)$ is a multiple of the identity $I$, and there exists a unitary operator $U$ and a $k$ th root $\lambda$ of 1 such that $\phi(A)=\lambda U A U^{*}$ for all $A \in \mathcal{B}(H)$.

It is natural and interesting to ask similar questions as in this paper for a more general case of the numerical radius.

Question 5.3. How do we classify the maps which preserve the numerical radius of operator products or operator Jordan triple-products?

\section{ACKNowledgments}

The authors wish to give their thanks to the referees for many valuable comments and suggestions. The authors also give their thanks to Chi-Kwong. Li and WaiShun Cheung for helpful discussions that simplified the original proof of (iii) $\Rightarrow$ (i) in Lemma 2.3.

\section{REFERENCES}

[1] Z.-F. Bai and J.-C. Hou, Z. B. Xu, Maps preserving numerical radius on $\mathrm{C}^{*}$-algebras, Studia Math., 162 (2004), 97-104. MR2046564 (2005a:47061)

[2] Z.-F. Bai and J.-C. Hou, Numerical radius distance preserving maps on $\mathcal{B}(H)$, Proc. Amer. Math. Soc., 132 (2004), 1453-1461. MR2053353(2005a:47060)

[3] J.-L. Cui and J.-C. Hou, Linear maps preserving the closure of numerical range on nest algebras with maximal atomic nest, Int. Equ. Oper. Theo., 46 (2003), 253-266. MR1991781 (2004k:47140)

[4] J.-L. Cui and J.-C. Hou, Non-linear numerical radius isometries on atomic nest algebras and diagonal algebras, J. Funct. Anal., 206 (2004), 414-448. MR2021854

[5] Jor-Jing Chan, Numerical radius preserving operators on $\mathcal{B}(H)$, Proc. Amer. Math. Soc., 123 (1995), 1437-1439. MR.1231293 (95f:47010)

[6] Jor-Jing Chan, Numerical radius preserving operators on $C^{*}$-algebras, Arch. Marh. (Basel), 70 (1998), 486-488. MR1621998 (99f:46078)

[7] Q.-H. Di , X.-F. Du and J.-C. Hou, Adjacency preserving maps on the space of hermitian operators, Chinese Ann. Math., to appear. 
[8] J.-C. Hou, Rank-preserving linear maps on $B(X)$, Sci. in China (Ser.A), 32 (1989), 929-940. MR:1055310 (92b:47052)

[9] C.-K. Li, P. Semrl and G. Soares, Linear operators preserving the numerical range (radius) on triangular matrices, Linear and Multilinear Algebra, 48 (2001), 281-292. MR1928398 (2003i:15024)

[10] L. Molnár, Some characterizations of the automorphisms of $\mathcal{B}(H)$ and $C(X)$, Proc. Amer. Math. Soc., 130 (2002), 111-120. MR1855627(2002m:47047)

[11] M. Omladic, On operators preserving the numerical range, Linear Algebra Appl. 134 (1990), 31-51. MR:1060008 (91i:47006)

[12] V. Pellegrini, Numerical range preserving operators on a Banach algebra, Studia Math, 54 (1975), 143-147. MR0388104 (52:8941)

[13] S. Pierce et.al., A survey of linear preserver problems, Linear and Multilinear Algebra 33 (1992), 1-129. MR 1346778

Department of Mathematics, Shanxi Teachers University, Linfen, 041004, People's Republic of China - and - Department of Mathematics, Shanxi University, Taiyuan, 030000, People's Republic of China

Department of Mathematics, Shanxi Teachers University, Linfen, 041004, People's Republic of China

E-mail address: jhou@dns.sxtu.edu.cn 